\title{
Amin Maalouf oder ein Schreiben zwischen Orient und Okzident
}

Während Elias Khoury aus der Perspektive der arabischen Literaturen auf den spanischsprachigen Roman des Kolumbianers Gabriel García Márquez in englischer Sprache ,antwortete،, entfaltete ein anderer Schriftsteller des vielsprachigen Libanon sein zwischen Orient und Okzident oszillierendes Schreiben in französischer Sprache. Ich spreche von Amin Maalouf - sicherlich einer der großen Autoren in französischer Sprache - und seinem Romanerstling Lèon l'Africain, der seit seinem Erscheinen aufhorchen ließ und ein romaneskes Oeuvre eröffnete, das in einer Vielzahl von Erzähltexten zwischen dem Nahen Osten und Europa, bisweilen - wie in seinem Roman Origines $^{1}$ - aber auch zwischen dem Nahen Osten, Frankreich und den Amerikas pendelt. Doch beschäftigen wir uns zunächst wie gewohnt mit einigen wenigen Biographemen, die uns einen Zugang $\mathrm{zu}$ diesem Vertreter der Literaturen des Libanon verschaffen sollen!

Amin Maalouf wurde am 25. Februar 1949 in der Nähe von Beirut geboren und lebt heute überwiegend in Paris sowie auf der kleinen französischen Atlantikinsel Ile d'Yeu, wo er in aller Ruhe schreiben kann. Bereits seine Herkunft verdeutlicht die komplizierten Verhältnisse im Libanon, gehören zu seinen Vorfahren doch ein presbyterianischer Prediger sowie ein katholischer Priester, und ist er selbst doch Sohn einer maronitischen Mutter und eines Vaters aus einer melkitischen Familie, die Verbindungen zum arabischen Stamm der Maalouf besitzt. Neben seiner arabischen Muttersprache erlernte er im Familienkreis auch die französische und englische Sprache. Als Melkit gehörte Maalouf zur christlichen Minderheit des Libanon. Sehr früh schon begriff er seine Rolle als Vermittler zwischen den Kulturen.

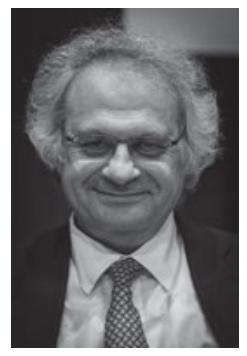

Abb. 121: Amin Maalouf (Nahe Beirut, 1949).

1 Vgl. hierzu Ette, Ottmar: Von Paris über Beirut nach Havanna. Transareale Reisebewegungen im literarischen Schaffen Amin Maaloufs. In: Klein, Wolfgang / Fähnders, Walter / Grewe, Andrea (Hg.): Dazwischen. Reisen_Metropolen_Avantgarden. Festschrift für Wolfgang Asholt. Bielefeld: Aisthesis Verlag 2009, S. 107-1

Ә Open Access. (C) 2021 Ottmar Ette, publiziert von De Gruyter. (c) BY-NC-ND Dieses Werk ist lizensiert unter einer Creative Commons Namensnennung - Nicht-kommerziell - Keine Bearbeitung 4.0 International Lizenz. https://doi.org/10.1515/9783110703450-036 
Er besuchte die französischsprachige Jesuitenschule Notre-Dame de Jamhour, wo er eine profunde Ausbildung erhielt und 1966 das französische sowie das libanesische Abitur ablegte. Ein Studium der Soziologie absolvierte er an der der Universität von Lyon angegliederten Ecole Supérieure des Lettres in Beirut. 1971 begann er nach dem Vorbild seines Vaters eine journalistische Laufbahn im Ressort für internationale Politik der arabischsprachigen libanesischen Tageszeitung An-Nahar (Der Tag) in Beirut. Doch dann kam die „Grande Guerre du Liban“ mit ihren Massakern und Morden, die auch für Maalouf einen biographischen Einschnitt bedeutete.

Wegen des Bürgerkriegs verließ Maalouf im Juni 1976 Beirut und ging über Zypern nach Paris. Von 1976 bis 1979 setzte er seine journalistische Laufbahn bei der Zeitschrift Jeune Afrique fort. Nach einem zweijährigen Intermezzo in den Jahren 1979 bis 1981 als Leiter der Pariser Redaktion von An-Nahar International kehrte er für die Zeit von 1981 bis 1985 zu Jeune Afrique als Chefredakteur zurück und veröffentlichte 1983 den viel beachteten historischen Essay Der Heilige Krieg der Barbaren. Die Kreuzzüge aus Sicht der Araber, in welchem er durch die Darstellung einer arabischen Sichtweise der christlichen Kreuzzüge zwischen Orient und Okzident zu vermitteln suchte. Damit war die Entscheidung für die Literatur und das schreiben in französischer Sprache gefallen: Bald schon erschien sein Debütroman Léon l'Africain, der 1986 zum Bestseller wurde.

In rascher Folge erschienen nun seine Romane, die sich stets mit dem Aufeinandertreffen verschiedener Religionen und Kulturen beschäftigten. Für seinen Roman Le Rocher de Tanios wurde er 1993 mit dem hochangesehenen Prix Goncourt ausgezeichnet. Im Februar 2000 ehrte das Weltwirtschaftsforum von Davos den Schriftsteller mit dem Crystal-Award, mit dem Maaloufs Engagement für Völkerverständigung und den Dialog der Kulturen gewürdigt wurde. In Buchessays wie Les Identités meurtrières setzte er sich mit grundlegenden Fragen des Zusammenlebens auseinander und prangerte einflussreich jegliche fundamentalistische Ausblendung kultureller Diversität als mörderisch und selbstmörderisch an. Amin Maalouf ist Mitglied der von Elie Wiesel gegründeten Académie Universelle des Cultures und setzte sich für eine Mehrsprachigkeits-Politik innerhalb der Europäischen Union ein. Maaloufs Werk wurde mit zahlreichen Preisen bedacht, er selbst mit der Ehrendoktorwürde der Universität Leuven ausgezeichnet.

Gerne will ich unseren kurzen Parcours durch einige Aspekte von Amin Maaloufs literarischem Werk mit seinem ersten Essay beginnen, Les croisades vues par les arabes, das die Perspektive auf die christlichen Kreuzzüge umkehrt, sich an der historiographischen Einteilung der arabischen Geschichtswissenschaft orientiert und damit einen Blickwechsel vornimmt, wie er für Maaloufs späteres romaneskes Schreiben charakteristisch ist. Es ist dieser Blickwechsel, der von 
einer monozentrischen Moderne des Okzidents abweicht und neue Perspektiven eröffnet, die literarisch ausgelotet werden können - ein Element, das man zweifellos einem Schreiben im Zeichen der Postmoderne zurechnen kann.

Mir ist es wichtig, dass Sie sich selbst einen kurzen Einblick in diese Sichtweise jahrhundertelangen Konflikts zwischen ,Abendland' und ,Morgenland“ verschaffen können, um die Aktualität dieses historischen Essays besser zu verstehen. Gerade auch aus einer gegenwärtigen Perspektive auf das Verhältnis zwischen Okzident und Orient, zwischen christlicher und islamischer Welt, ist es von grundlegender Bedeutung, immer wieder die Blickpunkte zu wechseln, um begreifen zu können, wie sehr sich im Zusammenspiel der Religionen Sichtweisen entfalteten, die das Andere als das Fremde als Erkenntnishorizont eigenen Verstehens ausschlossen.

Vergessen wir dabei nicht, dass sich lange nach Erstveröffentlichung dieses Bandes jener gefährliche Ausspruch des damaligen US-Präsidenten George W. Bush vom ,Kreuzzug‘ ansiedelt, den es gegen den Islam und die arabische Welt zu führen gelte, der ungeheuer viel an Bestürzung und zerstörerischem Potenzial auslöste. Ein Ausspruch freilich, der an Dummheit immer wieder vom aktuellen Präsidenten der Vereinigten Staaten von Amerika übertroffen worden ist. Die versöhnenden Gesten eines anderen Präsidenten, Barack Obama, konnten gegenüber derartigen Ausfällen kaum etwas bewirken - zu sehr war und ist im arabischen und islamischen Bewusstsein die Epoche der Kreuzzüge präsent und prägt das Bild, das man sich vom Okzident im Orient macht.

Die Gründe für eine derartige Haltung gegenüber dem ,Abendland' verstehen sie nach der Lektüre des Bandes von Amin Maalouf wesentlich besser. Ich möchte mit einer Passage aus dem Epilog von Les croisades vues par les arabes beginnen, wo Maalouf eine Art Bilanz kriegerischer Auseinandersetzungen und des scheinbaren Sieges der arabischen Welt über die Kreuzzügler vorlegt:

Dem Anschein nach erzielte die arabische Welt einen blendenden Sieg. Wenn das Abendland durch seine aufeinander folgenden Invasionen das Vordringen des Islam aufzuhalten suchte, dann war das Endergebnis das genaue Gegenteil. Denn nicht nur wurden die Kreuzzugsstaaten des Orients nach zwei Jahrhunderten der Kolonisierung mit der Wurzel ausgerissen, sondern die Muslime hatten sich so gut erholt, dass sie nun unter der Fahne der ottomanischen Türken wieder anstürmten und die Eroberung Europas selbst in Angriff nahmen. 1453 fällt Konstantinopel in ihre Hände. 1529 kampierten ihre Ritter unter den Mauern von Wien.

Aber dies war, wie wir sagten, nur der Anschein. Denn im Gefolge des historischen Rückzuges drängt sich eine Feststellung auf: In der Epoche der Kreuzzüge ist die arabische Welt, von Spanien bis hinüber zum Irak, auf der intellektuellen wie auf der materiellen Ebene noch die Hüterin der am weitesten fortgeschrittenen Zivilisation unseres Planeten. Danach aber wandert das Zentrum der Welt entschieden gen Westen. Besteht hier eine Verbindung zwischen Ursache und Wirkung? Darf man so weit gehen zu behaupten, dass die Kreuzzüge 
das Signal für den Aufstieg des westlichen Europa gaben - das Zug um Zug die ganze Welt beherrschen sollte - so wie sie das Totenglöcklein für die arabische Zivilisation läuteten?

Die Amin Maaloufs Schlussfolgerungen sind ebenso überraschend wie überzeugend. Denn nur an der Oberfläche habe der Orient durch die gescheiterten Invasionen der Kreuzzüge gewonnen und in der Folge das Oströmische Reich erobert, Konstantinopel oder Istanbul sowie die heute wieder zum Zankapfel zwischen Ost und West gewordene Hagia Sophia eingenommen, ja selbst die Hauptstädte Mitteleuropas bedroht. Ob man globalgeschichtlich die arabische Zivilisation als die damals fortgeschrittenste des ganzen Planeten bezeichnen darf, mag angesichts der chinesischen Zivilisation und den Zeichen des Glanzes von Tianxia umstritten sein. Tatsache ist aber, dass seit diesem Zeitpunkt der Aufstieg des westlichen Europa begann und es bald zur ersten Phase beschleunigter Globalisierung unter Führung Spaniens und Portugals kam - die beide zuvor die arabischen Reiche zurückgedrängt hatten. Der Abstieg der arabischen Welt hatte begonnen ...

Gewiss war Amin Maalouf bestrebt, eine derart schematische und vereinfachende Sichtweise der Weltgeschichte zu nuancieren. Doch erblickte er in der Zeit nach Ende der letzten Kreuzzüge einen historisch-intellektuellen Wendepunkt, dessen Fernwirkungen bis heute spürbar seien und der letztlich eine Asymmetrie der Macht zwischen Orient und Okzident befördert hätte. Dieses Ungleichgewicht hält bis heute an und werde sich - so die lange vor den Ereignissen des 11. September 2001 formulierte Hypothese - in Zukunft möglicherweise noch deutlich verstärken.

Folglich ist gerade der Schlussabschnitt dieses Epilogs zu den Kreuzzügen aus arabischer Sicht in vielerlei Hinsicht bemerkenswert und aufschlussreich. Nicht nur, weil das dort erwähnte Attentat auf den Führer der Christenheit durch den Tod Papst Johannes Pauls des Zweiten in den westlichen Medien wieder präsent war, sondern weil es zusammen mit einer ganz anderen, individuellen Dimension, mit der Gegengeste vordergründiger Versöhnung seitens des Papstes kommentiert und zirkuliert wurde. Kein Zweifel kann daran bestehen, dass die alten Bilder des Kampfs der Kreuzritter gegen die übermächtigen ,Sarazenen“ und die Bilder westlicher Invasoren, welche sich über Jahrhunderte der heiligen Stätten des Islam zu bemächtigen versuchten, gegenwärtiger und wirksamer sind, als wir uns alle dies eingestehen wollen. Nicht allein der Aufruf zum Kreuzzug durch einen US-amerikanischen Präsidenten, dem seine Berater und die Presse einflüstern mussten, dass er sich dieser Bilder bitte nicht noch einmal bedienen solle, sondern auch anti-westliche Rhetoriken islamischer Fundamentalisten

2 Maalouf, Amin: Les croisades vues par les Arabes. Paris: Jean-Claude Lattès 1983, S. 299. 
unterschiedlicher Färbung verraten auch heute noch viel von der Präsenz einer längst vergangenen Epoche.

Amin Maaloufs historiographischer Essay verrichtet ein gut Teil seiner Vermittlungsarbeit dadurch, dass er einen fundamentalen Blickwechsel inszeniert, durch welchen der Westen in die verschwiegenen Geschichtsversionen des Orients Einblicke erhält und letztlich auch umgekehrt Einsichten in die eigene Geschichte historischer Marginalisierung ermöglicht werden:

In einer ständigen Angriffen ausgesetzten muslimischen Welt ist die Herausbildung eines Gefühls der Verfolgung nicht zu verhindern, das bei gewissen Fanatikern die Form einer gefährlichen Obsession annimmt: Hat man am 13. Mai 1981 den Türken Mehmed Ali Agca nicht auf den Papst schießen sehen, nachdem er zuvor in einem Brief erklärt hatte: Ich habe mich entschlossen, Papst Johannes Paul II, den Obersten Heerführer der Kreuzzügler, zu töten. Jenseits dieses individuellen Aktes ist klar, dass der arabische Orient noch immer im Okzident einen natürlichen Feind erblickt. Gegen diesen ist jeder feindliche Akt, sei er politisch, militärisch oder auf dem Gebiet der Erdölförderung, nichts anderes als eine legitime Revanche. Und man kann nicht daran zweifeln, dass der völlige Bruch zwischen diesen beiden Welten von den Kreuzzügen her datiert, welche von den Arabern noch heute als eine Vergewaltigung empfunden werden. ${ }^{3}$

Ohne an dieser Stelle ausführlich auf diesen im Jahr 1983 erstmals veröffentlichten Essay eingehen zu können, führt uns Les croisades vues par les arabes doch in eine Weltsicht ein, die dafür plädiert, aus den einseitigen und vereinseitigenden Wahrnehmungsmustern herauszutreten, von denen wir im Verlauf der letzten Jahrzehnte auf beiden Seiten nicht wenige hervorgebracht haben. Die „self-fulfilling prophecy“ dieser antagonistischen Weltsichten ist in gewisser Weise der „Clash of Civilization“, der von Samuel P. Huntington ausgerufene „Kampf der Kulturen“. Im Gewand der Wissenschaft entstammt dieser Begriff und der dazugehörige Text der Feder eines Politologen, der zu den wichtigsten Beratern mehrerer Präsidenten der USA zählt, und gehört doch in keiner Weise - folgen wir den Überlegungen Peter V. Zimas ${ }^{4}$ - dem Bereich der Theorie und ihren Denkvoraussetzungen an, sondern ist ganz eindeutig dem Bereich der Ideologie zuzuschlagen. Aus diesen mehr oder minder stark fanatisierten Ideologien aber versucht die ruhige historiographische Arbeit Amin Maaloufs, in einem sachlichen und transparenten Stil abgefasst, herauszutreten und damit eine wichtige Vermittlungsarbeit zwischen Orient und Okzident zu leisten.

3 Maalouf, Amin: Les croisades vues par les Arabes, S. 304.

4 Vgl. Zima, Peter V.: Ideologie und Theorie. Eine Diskurskritik. Tübingen: A. Francke Verlag 1989. 
Drei Jahre nach dem Erfolg seines historischen Blickwechsels und seiner Entscheidung, dem Journalismus als Hauptberuf den Rücken zu kehren und sich nun ganz der Schriftstellerei zuzuwenden, erschien Maaloufs Erstlingsroman Lèon l'Africain, der seinen internationalen Ruf als Autor begründete. Titelfigur des als fiktive Autobiographie angelegten historischen Romans ist Johannes Leo Africanus alias Giovan Leone l'Africano alias Léon l'Africain. Es handelt sich bei ihm um den Verfasser einer um 1525 niedergeschriebenen und 1550 in Venedig von keinem Geringeren als Ramusio unter dem Titel Descrizione dell'Affrica $e$ delle cose notabili che quivi sono publizierten Schrift über Afrika, welche diesen Reisenden bis in Mungo Parks Zeiten in eine der wichtigsten Quellen hinsichtlich der afrikanischen Welt verwandelte. Da ich dieser spannenden historischen Figur ein Kapitel meiner Vorlesung ReiseSchreiben gewidmet habe, möchte ich Sie auf diesen Band der Reihe „Aula“ verweisen ${ }^{5}$ und mich in unserer jetzigen Vorlesung dem Roman des libanesischen Schriftstellers zuwenden.

Beginn und Struktur des Romans erinnern an die Gattungscharakteristika einer „Novela picaresca“, eines Schelmenromans also, denn der gut vierzigjährige Ich-Erzähler schildert in einer Art testamentarischem Brief an seinen Sohn, der ihm nachfolgen werde, einen überaus erstaunlichen Lebensweg, der zwischen Orient und Okzident oszillierte. Dabei spielten welthistorische Ereignisse eine wesentliche Rolle in seinem Leben, wurde der Werdegang des kleinen in Granada geborenen Jungen doch bereits durch die Eroberung der Stadt durch die „Reyes Católicos“ im Jahr 1492 wesentlich beeinflusst, da die Familie letztlich ins Exil nach Nordafrika getrieben wurde. Die sich anschließenden Ereignisse sind ebenfalls dem Leben des Johannes Leo Africanus nachempfunden, wobei es anzumerken gilt, dass Amin Maalouf durch seine literarische Rekonstruktion dieses Lebens eine längst vergessene Gestalt wieder aus dem Schatten holte und auch wissenschaftliche Forschungen und Veröffentlichungen zu dieser Figur seit Publikation des Romans deutlich zunahmen. Die diplomatischen Missionen und Handelsreisen, während derer der Protagonist mit dem noch arabischen Namen al-Hassan al-Wazzan teilweise in die Rolle eines Forschungsreisenden schlüpft und nicht selten auch in die des Vermittlers zwischen den Kulturen, führen ihn quer durch die Sahara zum glanzvollen Timbuktu sowie in mehrere subsaharische Königreiche und schließlich bis nach Konstantinopel. Nach zahlreichen, zum Teil abenteuerlichen Erlebnissen erfährt sein Leben bei der Rückkehr von einer Pilgerfahrt nach Mekka jedoch eine entscheidende Wendung.

5 Vgl. Ette, Ottmar: ReiseSchreiben. Potsdamer Vorlesungen zur Reiseliteratur. Berlin - Boston: Walter de Gruyter 2020, S. 319-340. 
Denn der gebildete, sprachgewandte und weltoffene Muslim wird von spanischen Korsaren unter Pedro de Bobadilla entführt und dem Medici-Papst Leo X. als Sklave geschenkt. Dieser weiß die intellektuellen Qualitäten Hassans zu schätzen und macht ihn zu seinem Lieblingsdiener, dem er weitreichende Freiheiten gibt und mit dem Auftrag ausstattet, sein Wissen in Buchform und in einer der von ihm erst kürzlich erlernten europäischen Sprachen niederzuschreiben. Doch zuvor wird der junge Granadiner als Symbol des Sieges der Christenheit über den Islam vor versammeltem diplomatischem Corps im Vatikan vom Papst höchstselbst auf den Namen Johannes Leo getauft.

Nach seinem wohl erzwungenen Übertritt zum christlichen Glauben unterrichtet man den jungen Mann, von dem wir möglicherweise ein Portrait besitzen, in der lateinischen, hebräischen, italienischen und türkischen Sprache. Unter christlichem Taufnamen entsteht sein großes Buch über Afrika, das für Jahrhunderte die bevorzugte Quelle europäischen Wissens über den gesamten Kontinent darstellen sollte. Der Angriff der Soldateska Karls V. auf die päpstliche Stadt, welcher unter der Bezeichnung „Sacco di Roma“ in die Geschichtsbücher einging, setzt schließlich seinem Aufenthalt am Tiber ein Ende. Er weicht in den Norden Italiens aus, schließt sein großes Afrika-Werk ab und tauscht ein weiteres Mal die Nordseite des Mittelmeeres mit dessen Südseite, indem er Richtung Tunis aufbricht. Nach der Überfahrt Richtung Tunesien verlieren sich ebenso die Spuren von Johannes Leo Africanus wie die der literarischen Gestalt in Amin Maaloufs beeindruckendem historischem Roman.

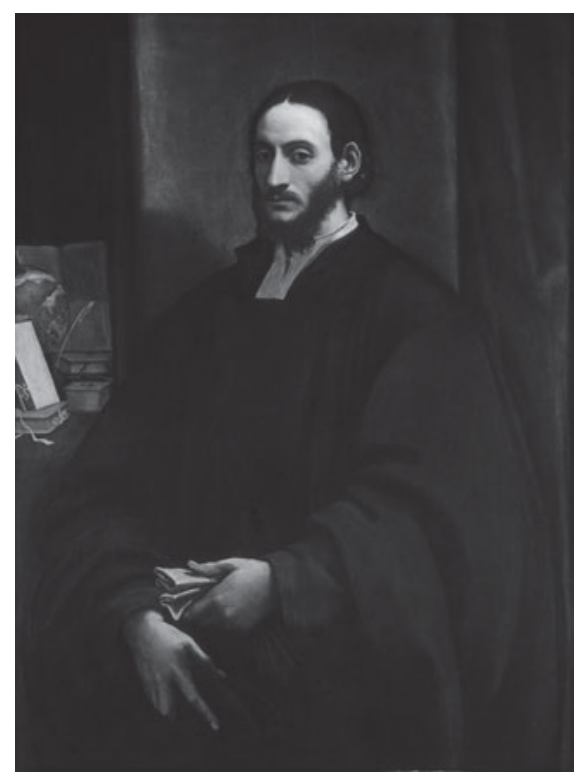

Abb. 122: Sebastiano del Piombo: Porträt eines Humanisten, angeblich Porträt des Johannes Leo Africanus, Öl auf Leinwand, 1529. 
Vor allem beeindruckt, mit welch spielerischer Leichtigkeit der libanesische Autor mit seinem Protagonisten umgeht, ihm fiktive Gestalten an die Seite stellt und ihn zu einer Art alter Ego modelliert. Amin Maalouf orientiert sich an historischen Fakten, lässt seiner Fantasie aber immer wieder freien Lauf und erreicht auf diese Weise eine Erzähldichte, in welcher wir dem jungen al-Hassan al-Wazzan wie dem älter gewordenen Johannes Leo Africanus gerne durch seine Abenteuer folgen. Wir fühlen uns in unserer Vorlesung erinnert an jene Arbeit des kubanischen Autors Reinaldo Arenas an seiner historischen Figur Fray Servando Teresa de Mier, welche ebenfalls von einer starken Orientierung an den intertextuellen Bezügen bestimmt war. Dabei schien freilich eine große Gestaltungsfreiheit auf und eine Vielzahl literarischer Verfremdungseffekte wurde eingeführt, die in Amin Maaloufs um historische Glaubwürdigkeit bemühten Roman größtenteils fehlen. Léon l'Africain wagt auf der formalen Ebene seines Erzählens weit weniger und erzielt mit Hilfe einer gediegenen Erzählmaschinerie, die sich noch an seinem wenige Jahre zuvor veröffentlichten historischen Essay ausrichtet, einen riesigen Verkaufserfolg, welcher selbst noch die Rezeption seiner nachfolgenden Romane wesentlich bestimmen sollte. Nicht umsonst ist Amin Maalouf in etwa dreißig verschiedene Sprachen weltweit übersetzt.

Al-Hassan al-Wazzan ist von Beginn des Romans an ein Wanderer zwischen den Welten, der mit größter kultureller Offenheit allem Fremden vorurteilslos begegnet und es wenn nötig auch in sein eigenes Leben inkorporiert. Maalouf gelingt es ausgezeichnet, diese Mobilität und mehr noch Vektorizität in sein Romangeschehen $\mathrm{zu}$ integrieren. ${ }^{6}$ Seine Position ist nicht nur die eines interkulturellen Übersetzens zwischen den Kulturen, sondern einer transkulturellen Querung unterschiedlichster kultureller Prägungen, was ihn als literarischen Helden zur Zentralfigur eines Schreibens im Zeichen der Postmoderne macht. Gerade in der Auseinandersetzung mit einem um Zentralisierung, Homogenität und Herrschaft bemühten Christentum erweist sich die vom realen Autor Amin Maalouf in die Gestalt eingeführte Flexibilität und Mobilität als Modell eines transkulturellen Verhaltens, das in einer sich verstärkt globalisierenden Welt vonnöten ist.

Selbstverständlich spielt Amin Maalouf hier mit bestimmten Anachronismen, die er aber immer wieder sanft ins Gedächtnis ruft und damit seiner Leserschaft verdeutlicht, dass es nicht allein um historische Positionen, sondern auch um

6 Vgl. zu dieser Vektorizität Ette, Ottmar: „Ma patrie est caravane“. Amin Maalouf, die Frage des Exils und das ZusammenLebenswissen der Literaturen ohne festen Wohnsitz. In: Romanistische Zeitschrift für Literaturgeschichte / Cahiers d'Histoire des Littératures Romanes (Heidelberg) XXXII, 3-4 (2009), S. 413-445. 
ein transkulturelles Vermittlungsverhalten zwischen Orient und Okzident in der Gegenwart geht. Denn die Vorstellung eines Wanderers zwischen den Kulturen bezieht sich nicht allein auf den jungen Granadiner, sondern auch auf den damals noch jungen libanesischen Schriftsteller selbst. Zugleich tritt Maalouf gerade in seinem historischen Rückblick gegen jegliche rückwärtsgewandte Sichtweise des Eigenen oder der eigenen Religion ein und lässt anhand der Figur seines zwischen den Kulturen sich frei bewegenden Protagonisten erkennen, wie sehr er auf die künftige, schon bald bevorstehende Macht einer kulturellen Verständigung zwischen Abendland und Morgenland vertraut. Dies ist ein Optimismus, den man auch noch seinem wunderbaren Essayband Les Identités meurtrières anmerkt, eine kulturhistorische Zuversicht, die in seinen späteren Schriften aber immer mehr einer skeptischeren Sichtweise Platz macht. Amin Maalouf musste den Begleiterscheinungen der vierten Phase beschleunigter Globalisierung wie dem ausgerufenen Kampf der Kulturen, den Fundamentalismen jeglicher Couleur und dem deutlichen Rechtsruck vieler Gesellschaften weltweit in seinem damals noch optimistischen Glauben an den kulturellen Fortschritt leider erheblichen Tribut zollen. Doch bleiben wir bei Léon l'Africain, einem zirkum-mediterranen Roman, der das Mittelmeer als Begegnungsraum unterschiedlichster Kulturen in den Mittelpunkt rückt.

In seinem zurecht mit Preisen überhäuften Roman findet sich eine charakteristische Vielsprachigkeit, indem in den französischen Text Elemente anderer Sprachen und insbesondere des Arabischen eingearbeitet wurden. Diese polyglotte und zugleich transkulturelle Dimension ist bereits im berühmten Incipit präsent, in dem sich - wie in jedem herausragenden literarischen Text - jene zentralen Isotopien auffinden lassen, die den gesamten Erzähltext durchqueren und strukturieren:

Ich, Hassan, Sohn von Mohammed, dem Wagner, ich, Johann Leo von Medici, beschnitten von der Hand eines Barbiers und getauft von der Hand eines Papstes, werde heute der Afrikaner genannt, doch komme ich nicht aus Afrika, noch aus Europa oder Arabien. Man nennt mich auch den Granadiner, den Fassi, den Zayyati, doch bin ich aus keinem Land, aus keiner Stadt, von keinem Stamme. Ich bin ein Sohn der Straße, meine Heimat heißt Karawane, und mein Leben ist die unerwartetste aller Durchquerungen.

Meine Hände haben Stück für Stück die Liebkosungen der Seide und die Beleidigungen der Wolle, das Gold der Fürsten und die Ketten der Sklaven kennen gelernt. Meine Finger haben tausend Schleier gelüftet, meine Lippen tausend Jungfrauen erröten lassen, meine Augen haben Städte sterben und Reiche untergehen sehen.

Aus meinem Munde kannst Du Arabisch, Türkisch, Kastilisch, das Berberische und Hebräische, das Lateinische und die italienische Volkssprache vernehmen, denn alle Sprachen, alle Gebete gehören zu mir. Ich dagegen bin von nichts und niemandem. Ich gehöre nur Gott und der Erde, und zu beiden werde ich eines nicht mehr fernen Tages zurückkehren. 


\begin{abstract}
Und nach mir wirst Du bleiben, mein Sohn. Und Du wirst mein Andenken bewahren. Und Du wirst meine Bücher lesen. Und dann wird Dir wieder jene Szene vor Augen treten: Dein Vater in neapolitanischer Kleidung auf einer Galeere, die ihn zurück zur afrikanischen Küste bringt, eifrig vor sich hin kritzelnd, einem Händler gleich, der am Ende einer langen Reise Bilanz zieht.

Aber ist dies nicht, was ich auch gerade tue: Was habe ich gewonnen, was verloren, was werde ich dem höchsten Gläubiger sagen? Vierzig Jahre hat er mir gewährt, die ich zugebracht habe, wohin die Reise mich führte: Meine Weisheit hat in Rom gelebt, meine Leidenschaft in Kairo, meine Angst in Fez, und in Granada lebt noch immer meine Unschuld. ${ }^{7}$
\end{abstract}

Sie haben damit die gesamte erste Seite des Romans vor sich, die dem Ablauf der nachfolgenden vier Bücher - welche hier im letzten Abschnitt des Zitats eingespielt werden - vorangestellt ist. Es ist in nuce der gesamte Roman. Wir können bereits an dieser Stelle sagen, dass im Zentrum des Romans die Reisebewegungen, ja alle möglichen Arten von Bewegungen und Bewegungsmustern stehen. Denn es sind Bewegungen im Raum, welche den Raum für Begegnungen schaffen.

Dabei wäre es ein Leichtes aufzuzeigen, dass dieser Darstellung eine gleichsam transtemporale Grundstruktur als Ausgangspunkt dient: Die historische Figur al-Hassan al-Wazzan vom Ende des 15. und Anfang des 16. Jahrhunderts wird im Alter von etwa vierzig Jahren und als Granadiner in sein Exilland nach Nordafrika zurückkehrend porträtiert. Aber zugleich werden transtemporal Elemente der Figur - und ich meine das durchaus als Bewegungsfigur - eines Intellektuellen erkennbar, der ebenfalls ein „fils de la route“, ein Sohn der Straße ist, keinem einzelnen Land zugeordnet oder zugeschlagen werden kann und dessen „patrie“ eben die „caravane“ ist, eine immerwährende Bewegung. Man könnte mit Blick auf den Titel eines der aussagekräftigsten Werke der deutschsprachigen Literaturen ohne festen Wohnsitz sagen, dass für den Protagonisten von Amin Maalouf der Roman Das Leben ist eine Karawanserei von Emine Sevgi Özdamar wie die Faust aufs Auge passt. Und in der Tat ist es ein translinguales Schreiben jenseits der eigenen Muttersprache, das nicht allein dasjenige der in der Türkei geborenen deutschsprachigen Autorin, sondern auch jenes des im Libanon geborenen französischsprachigen Schriftstellers charakterisiert. Wir haben es folglich mit einem Beispiel für die Literaturen ohne festen Wohnsitz zu tun, die - wie wir sahen - einen wichtigen und stets wachsenden Anteil an den Literaturen der Welt einnehmen.

In subtiler, aber durchaus kenntlich gemachter Weise wird hier mit Blick auf Amin Maalouf, den realen Autor selbst, eine Dimension sichtbar, die man als Schreiben ohne festen Wohnsitz in französischer Sprache bezeichnen kann,

7 Maalouf, Amin: Léon l’Africain. Paris: Jean-Claude Lattès 1986, S. 9. 
einem Schreiben, das von Beginn an die Spuren verschiedenster Sprachen trägt. Nicht einem bestimmten Ort, einer bestimmten Area, nicht einer bestimmten Nationalliteratur ist dieses Schreiben, dieses Gekritzel zuzuordnen, sondern einer Tätigkeit des fortgesetzten Reisens, der immerwährenden Ortsveränderung, der nie enden wollenden Mobilität, die alle Seiten dieses Erzähltextes mit ihren ständigen Blickwechseln prägt. Nicht umsonst entsteht das „griffonner“, das Kritzeln auf den Seiten, die sich explizit an den eigenen Sohn und damit die Nachkommenschaft insgesamt wenden, an Bord eines Schiffes: auf hoher See zwischen den beiden Welten, die das Leben des Ich bestimmt haben: zwischen Orient und Okzident.

Zugleich wird auch deutlich, dass dieses Ich die verschiedensten Sprachen quert und spricht, sich folglich in einer translingualen Situation befindet, welche in der Tat ebenfalls auf beiden Zeitebenen - der des 16. wie der des ausgehenden 20. Jahrhunderts - eine prägende Rolle spielt. Dieses Queren verschiedener Sprachen hat ohne jeden Zweifel mit der Tatsache zu tun, dass Amin Maalouf selbst sich ja in einer Sprache als Literatursprache ausdrückt, die er zwar früh schon lernte und in seiner Ausbildung anwandte, die aber nicht seine eigene Muttersprache ist. Das Pendeln zwischen den Sprachen erst baut jenes vielgestaltige Territorium des Mittelmeeres auf, dessen transmediterrane Dimension ${ }^{8}$ ebenso sprachlich wie kulturell allgegenwärtig ist. Das Spannungsfeld dieses Bewegungsraums wird in Maaloufs Roman von den Städten Granada, Fes, Rom, Kairo und Konstantinopel alias Istanbul gebildet. Wenn wir eine autobiographische Lesart akzeptieren, ohne sie freilich zur einzigen oder auch nur zur dominanten machen $\mathrm{zu}$ wollen, so dürfen wir feststellen, dass sich hier Bewegungsmuster und vektorielle Speicherungen situieren, die mit Blick auf Amin Maaloufs Gesamtwerk von höchster Bedeutung sind. Und dies ebenso wie sie auch für unser Verständnis der Romanischen Literaturen der Welt in jener historischen Tiefenschärfe, die unsere Arbeit und unsere transarealen Konzeptionen leitet, eminente Relevanz besitzen.

Amin Maalouf hat mit Bedacht diese Epoche gewählt und bewusst transareal ausgestaltet. Denn es handelt sich just um eine Zeit, die wir als die „Erste Phase beschleunigter Globalisierung“ bezeichnen dürfen, in der grundlegende Umgestaltungen von weltumspannender Bedeutung stattfanden. Denn im „annus mirabilis“ 1492, das kurz nach der Geburt des kleinen al-Hassan al-Wazzan lag,

8 Vgl. hierzu Hofmann, Franck / Messling, Markus: Die Krise Europas. Von Gide bis Godard: Denken der Méditerrannée und der europäischen Kultur. In: Lendemains (Tübingen) XXXVIII, 150 (2013), S. 141-152; dies. (Hg.): Leeres Zentrum. Das Mittelmeer und die literarische Moderne. Eine Anthologie. Berlin: Kulturverlag Kadmos 2015; sowie dies. (Hg.): Point de fuite. La Méditerrannée et la Crise Européenne. Berlin: Kadmos 2019. 
liefen die verschiedensten globalgeschichtlichen Linien zusammen und von hier aus gingen die unterschiedlichsten geschichtlichen Prozesse aus, die zum Teil noch heute nicht $\mathrm{zu}$ ihrem Abschluss gekommen sind. Zum einen fiel 1492 die Stadt Granada, was die Beseitigung der letzten arabischen Herrschaft auf iberischem Boden bedeutete, womit eine sieben Jahrhunderte andauernde Dominanz arabischer Macht und großer kultureller Blüte in Iberien zu Ende ging. Ein historischer Zyklus war damit abgeschlossen, der im Jahr 711 mit dem Übersetzen der Truppen des Tāriq in der Nähe des heute nach ihm benannten Felsen von Gibraltar begonnen hatte.

Zweitens signalisiert das Jahr 1492 - wie Sie wissen - die sogenannte ,Entdeckung' der Neuen Welt. Dabei ereignete sich in der Stadt Granada, die gerade von den Katholischen Königen eingenommen worden war, beziehungsweise im nahe gelegenen Santa Fe jene Begegnung zwischen Cristoforo Colombo alias Cristóbal Colón - ebenfalls einem wahren „transméditerranéen“ - und Königin Isabella von Kastilien, welche die Grundlagen für das Ausgreifen der iberischen Mächte nach Amerika legte. Die Ergebnisse dieser Begegnung prägten eine Vielzahl sich anschließender Ereignisse, welche über ein Gutteil der kommenden Menschheitsgeschichte bestimmte.

Drittens markiert das Jahr 1492 mit der Ausweisung der Juden aus dem gesamten Königreich der Katholischen Könige einen - wenn Sie mir den Ausdruck gestatten - ,christlichen Fundamentalismus', insofern der Alleinvertretungsanspruch des Katholizismus, der bereits in diesem Begriff selbst präsent ist, mit Feuer und Schwert durchgeführt werden sollte. Die Einführung einer zentralisierten Macht und eines kulturell wie ethnisch homogenisierten Raumes schufen Grundlagen dessen, was wir als „Europäische Moderne“ bezeichnen dürfen: Nicht umsonst beginnt Ende des 15. Jahrhunderts jene Epoche, die in verschiedenen europäischen Sprachen als „Modern Times“, als „Les temps modernes“, als „Los tiempos modernos“ oder auch als „Neuzeit“ bezeichnet wird. Die Wahl, vor welche die Juden, aber auch andere Minderheiten im spanischen Königreich gestellt wurden, war einfach: entweder Konvertierung zum christlichen Glauben (unter weiterer Beobachtung durch die Heilige Inquisition) oder sofortige Vertreibung aus Spanien beziehungsweise Tod.

Schließlich ist das Jahr 1492 auch mit der Entstehung der ersten modernen Grammatik durch Nebrija und mit einer sprachlich-linguistischen Dimension der Expansion verbunden. Ganz im Sinne Nebrijas beinhaltete letztere die geistige Engführung von Sprache und Herrschaft, folglich eine direkte Verbindung zwischen dem Kastilischen oder Spanischen und dessen Verbreitung unter den eroberten Völkern, zwischen Expansion des Reiches und Expansion der Sprache als geordneter Herrschaftssprache innerhalb eines entstehenden Imperiums, das als zentralisierter, homogener Machtraum gedacht wurde. Damit begann jener 
kontrollierte Prozess und Siegeszug, der das Spanische in eine Weltsprache verwandeln sollte. Auf vielen verschiedenen Ebenen markiert das Jahr 1492 damit einen Prozess, in dem sich mit ungeheurer Geschwindigkeit und Wucht die erste Phase beschleunigter Globalisierung entwickeln sollte.

Amin Maalouf waren all diese globalgeschichtlich bedeutsamen Ereignisse bestens vertraut. So konnte er der Versuchung nicht widerstehen, diese entscheidende geschichtliche Wende mit dem Fall Granadas, der Vertreibung der Juden und der Öffnung auf eine Neue Welt, letztlich einen Expansionsprozess des westlichen Europa, auf den er bereits in seinem Epilog zu Les croisades vues par les arabes hingewiesen hatte, in seine Romandiegese einzubauen. Ich möchte Ihnen diese romantechnisch geschickt eingefädelte Passage nicht vorenthalten, in der eine Verbindung zwischen den „caravanes“ und den „caravelles“ hergestellt wird:

In der Tat ging das Leben im besetzten Granada unmittelbar weiter, so als ob Ferdinand vermeiden wollte, dass die Muslime in Massen ins Exil strömten. Die Geiseln kehrten gleich am Tag nach dem Einzug des Königs und der Königin in die Stadt zu ihren Familien zurück, und mein Vater erzählte uns, dass er mit größeren Aufmerksamkeiten als ein Gast im Prinzenrang behandelt worden sei. In Santa Fé waren seine Gefährten und er keineswegs in ein Gefängnis eingesperrt; sie konnten vielmehr den Markt besuchen und bisweilen in kleinen Gruppen durch die Straßen spazieren, freilich begleitet von Wachen, welche die Aufgabe hatten, sie gleichzeitig zu überwachen und vor den Wutausbrüchen manches betrunkenen oder erregten Soldaten zu schützen. Im Verlaufe eines dieser Rundgänge zeigte man meinem Vater einen Seefahrer aus Genua, der in der Tür einer Taverne stand und von dem ganz Santa Fé sprach und ihn belächelte. Man nannte ihn „Cristóbal Colón“. Er wollte, so sagte er, Karavellen ausrüsten, um Indien über den Westen zu erreichen, denn die Erde sei rund, und er verbarg nicht seine Hoffnung, für diese Expedition einen Teil des Schatzes der Alhambra zu erhalten. Er befand sich schon seit Wochen vor Ort und bestand darauf, den König oder die Königin zu treffen, welche ihn freilich mieden, obwohl er ihnen von hohen Persönlichkeiten empfohlen worden war. Beim Warten auf eine Audienz schrieb er ihnen pausenlos Botschaften und Bittschriften, was beide in diesen Kriegszeiten überaus störte. Mohammed sah diesen Genuesen niemals wieder, aber ich selbst hatte noch oft die Gelegenheit, von ihm sprechen zu hören. ${ }^{9}$

An die Stelle der Karawanen treten hier die Karavellen, an die der „Reconquista“ iberischer Erde erscheint am Horizont die „Conquista“ einer Neuen Welt. In dieser Passage wird auf wenigen Zeilen die zeitgenössische Situation der Expansion Spaniens auf dem Gebiet der Iberischen Halbinsel mit der historischen Expansionsbewegung der iberischen Mächte so miteinander verbunden, dass das Ich von Maaloufs Roman sich gleichsam innerhalb dieses raum-zeitlichen Horizonts oder Spielraums bewegt. Wir befinden uns an einer zentralen Wegkreuzung der

9 Maalouf, Amin: Léon l’Africain, S. 66. 
Weltgeschichte, insofern sich die Aufmerksamkeit Spaniens fortan stärker nach Westen wendet und für Europa ein neues Zeitalter heraufzieht. Léon l'Africain wird ein wichtiger Zeuge dieses neuen Zeitalters sein und sein Bericht über Afrika gemeinsam mit den ersten Berichten über die Neue Welt in jene Kollektionen und Sammlungen eingehen, die das europäische Wissen und die europäische Herrschaft über weite Teile des Planeten begründeten.

Damit wird zugleich eine geschichtliche, politische, wirtschaftliche und kulturelle Konstellation skizziert, innerhalb derer sich die Reisen von al-Hassan alWazzan durch den afrikanischen Kontinent in einer Beziehung zur Expansion der christlichen Länder stehen, die für die von Maalouf behandelte Epoche charakteristisch ist. Es ist historisch mehr als zutreffend, dass die „Reconquista“ auch auf afrikanischem Territorium bald in eine „Conquista“ übergehen sollte, in eine Eroberungspolitik nicht nur jenseits der Säulen des Herkules, sondern auch entlang der afrikanischen Küste. Diese Phase der Eroberungen findet noch heute in Städten wie Ceuta oder Melilla ihren Ausdruck und erinnert uns daran, dass diese Phase keineswegs vergessen ist. Die Wunden scheinbar längst vergangener Kriege und Konflikte liegen in unseren Landkarten offen zutage. Kein Zweifel: Das Romangeschehen situiert sich innerhalb der ersten Phase beschleunigter Globalisierung, welche sich in wesentlicher Weise auf militärischem, aber auch auf technologischem und kulturellem Gebiet in einer Vielfalt an Phänomenen und Ausdrucksformen zeigte, die Maaloufs Roman gekonnt in seine verdichtete Fiktion einzubinden verstand. Sein Roman macht uns darauf aufmerksam, dass nicht allein der Westen Europas, sondern die gesamte Alte Welt in einen historischen Beschleunigungsprozess eingebunden wurde, der die politischen wie kulturellen Beziehungen zwischen allen Weltteilen von Grund auf veränderte.

Der Blick auf die geschichtliche Situation Ende des fünfzehnten und Anfang des sechzehnten Jahrhunderts zeigt uns eine Welt im Wandel. Das letzte Nasriden-Reich auf der iberischen Halbinsel ist zusammengebrochen, doch gleichzeitig rücken mit fast unwiderstehlicher Kraft die Türken im Osten des Mittelmeerraumes auf dem Balkan vor und werden kurze Zeit später vor den Toren Wiens stehen. Während sich Europa nach Westen, nach Amerika hin ausdehnt, werden noch immer die türkischen Muslime selbst die Kernbereiche Mitteleuropas bedrohen. Es ist aufschlussreich, wie sich die Präsenz ebenso Amerikas wie auch der islamischen Welt und ihrer Übergangsformen gerade auch an den Höfen der christlichen Herrscher Europas zeigte.

Es bleibt sicherlich festzuhalten, dass man - ähnlich, wie dies die Spanier in der Neuen Welt, etwa in Neuspanien oder Mexiko, taten - sehr gerne die Söhne herausragender Männer aus dem gegnerischen Lager zu gewinnen, zu taufen und umzuerziehen versuchte, um sie als mögliche künftige Führungselite in den von Christen eroberten Regionen als politische Marionettenfiguren 
in der Hinterhand zu halten. Die Unterschrift eines Fürsten von Fez am 30. April 1508 im Gefolge Kaiser Maximilians, aber auch viele andere Zeugnisse künden von der Wichtigkeit und der Präsenz dieser Mauren an christlichen Höfen und in christlichen Reichsvorstellungen. Es handelt sich um denselben Maximilian, der zusammen mit einem tanzenden Mauren auf dem Goldenen Dachl zu Innsbruck dargestellt wurde, vor dem ich einst als kleiner Junge staunend stand. Es sind Jahrzehnte einer intensiven Konfrontation zwischen Orient und Okzident, Islam und Christentum, in welche sich selbstverständlich auch die Taufe al-Hassan alWazzans durch keinen Geringeren als den Medici-Papst am Jahrestag des Einzugs der Katholischen Könige in die eroberte Stadt Granada einfügt. Amin Maalouf baut diese geschichtlichen Hintergründe immer wieder geschickt in seinen historischen, aber an Fiktionen reichen Roman ein.

Gestatten Sie mir noch bitte einen kurzen Seitenblick auf das eben erwähnte Goldene Dachl, das mich immer schon faszinierte und das ein besonderes Licht auf jenen Zeitraum wirft, mit dem wir es jetzt zu tun haben. Es handelt sich dabei um den Bau oder Umbau eines Prunkerkers in der Stadt Innsbruck, der zwischen den Jahren 1494 und 1496 vorgenommen wurde. Kaiser Maximilian ließ anlässlich seiner (zweiten) Heirat mit Bianca Maria Sforza von Mailand nicht nur alle Insignien der Macht an diesem Goldenen ,Dach` anbringen, sondern auch Fresken und Reliefs mit sogenannten Moriskentänzern ausführen, die seine zentral gestellte Gestalt - und die seiner beiden Frauen - der aktuellen wie der verstorbenen gleichsam rahmen.
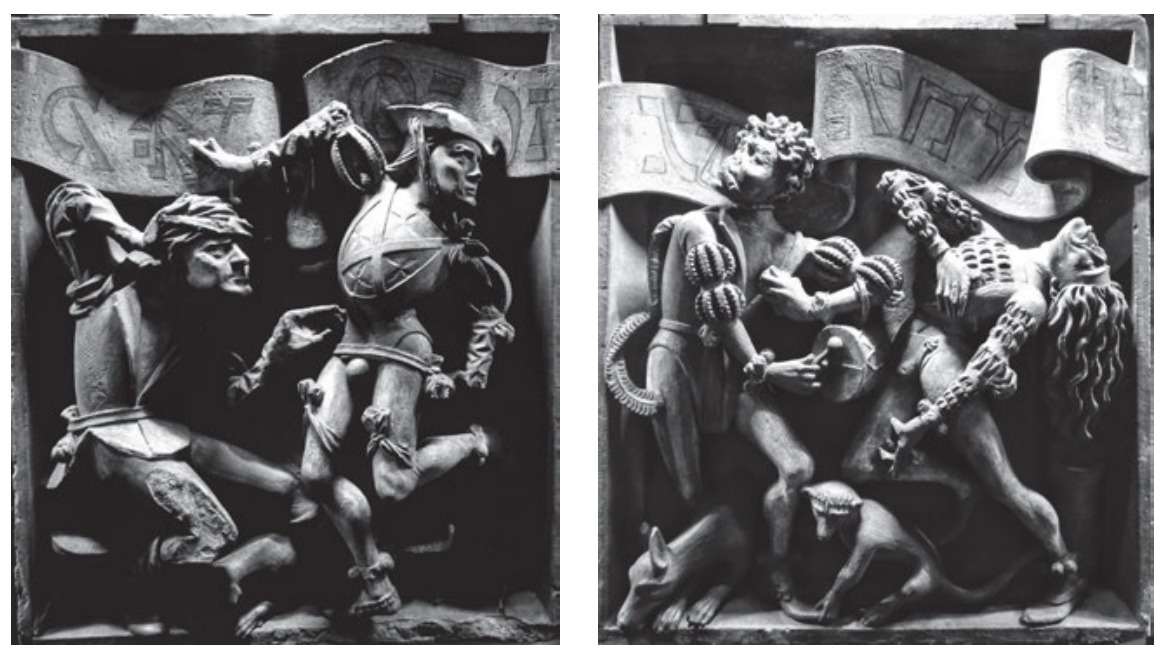

Abb. 123a und b: Reliefdarstellung zweier Moriskentänzer am Goldenen Dachl in Innsbruck, 1497-1500. 
Diese Darstellung von Nicht-Christen sollte die beherrschende Stellung der Christenheit und christlicher Reiche zu einem Zeitpunkt unterstreichen, als die Alhambra und das Nasriden-Reich von Granada gefallen waren und zugleich der Sprung nach Afrika angegangen werden sollte. Nicht umsonst rief der MediciPapst Leo X. aber schon wenige Jahre später angesichts der Bedrohung durch das Osmanische Reich - wenn auch erfolglos - zu einem neuerlichen Kreuzzug auf. Es handelt sich um just jenen Papst, der durch eine Erneuerung des Ablasshandels zur Finanzierung des Weiterbaus der Peterskirche in Rom den Anstoß zur Reformation gab: Wir sind also in historisch wie religiös äußerst bewegten Zeiten. $\mathrm{Zu}$ einem erneuten Kreuzzug kam es jedoch nicht mehr. Jedenfalls war die Sympathie Kaiser Maximilians für den Moriskentanz zu einer Zeit intensiver Kriege und Kämpfe weithin bekannt. Als er im Jahr 1492 als König die Stadt Konstanz besuchte, war der damalige Magistrat über diese Vorliebe des Kaisers wohlinformiert. $\mathrm{Zu}$ seiner Freude nämlich wurde von den schwarz bemalten Gesellen der Fischerzunft 1492 ein sogenannter „Mohrentanz“ aufgeführt, mit den man den Herrscher in den Konstanzer Stadtmauern begrüßte. Soviel als kleiner, aber hoffentlich anschaulicher Seitenblick auf eine historische Figur, welche in diesen von Maalouf geschilderten Zeitraum rund um Johannes Leo Africanus passt.

Amin Maalouf hat seinem Roman eine Widmung vorangestellt, die ein Zitat des irischen Dichters Butler Yeats enthält: Man solle nicht daran zweifeln, dass dieser Leo Africanus letztlich er selbst - „ich selbst“ - sei. Die damit eingeführte autobiographische Dimension, die zugleich eine friktionale Dimension mit Blick auf sehr unterschiedliche Diegesen enthält, bezieht sich bei Maalouf auf das Schwanken, das Oszillieren, die Bewegung zwischen Orient und Okzident. Immer wieder war Maalouf für die französische Regierung auf Vermittlungsmission im Nahen Osten tätig.

Wir haben die erste Seite des Romans ausführlicher analysiert und auf ihre Qualität als ZwischenWeltenSchreiben hin untersucht. Nun möchte ich Ihnen gerne zum Abschluss unserer Beschäftigung mit Léon l'Africain die letzte Seite von Maaloufs historischem Roman vorstellen, denn sie entwickelt etwas, das bis heute im Dunkeln blieb. Wir haben in der Tat keinerlei Zeugnisse mehr von alHassan al-Wazzan nach seiner ,Rückkehr` in den nordafrikanischen Raum nach Tunis: Hier brechen alle glaubwürdigen Berichte über den Granadiner ab. Amin Maalouf imaginiert eine letzte Seite, die von der Seite Tunesiens her - und damit nicht von der Granadas - das bisherige und zu Ende gehende „périple“, die Wege und Reisen des Johannes Leo Africanus, bilanziert. Schauen wir uns diesen Schlusspunkt des Texts genauer an: 
Ein letztes Wort auf die letzte Seite geschrieben, schon an der afrikanischen Küste.

Weiße Minarette von Gammarth, ehrwürdige Ruinen von Karthago, in ihrem Schatten bedroht mich das Vergessen, hin zu ihnen entgleitet nach so vielen Schiffbrüchen mein Leben. Die Plünderung Roms nach der Geißelung Kairos, das Feuer von Timbuktu nach dem Fall von Granada: Ist es das Unglück, das mich ruft, oder bin ich es wohl, der das Unglück ruft?

Einmal mehr, mein Sohn, werde ich von diesem Meer getragen, als Zeuge all meiner Irrungen, es ruft Dich gegenwärtig in Dein erstes Exil. In Rom warst Du „der Sohn des Afrikaners“; in Afrika wirst Du „der Sohn des Römers“ sein. Wo auch immer Du sein magst, werden Menschen Deine Haut und Deine Gebete durchsuchen. Hüte Dich, ihren Instinkten zu schmeicheln, mein Sohn, hüte Dich, unter der Menge nachzugeben! Muslim, Jude oder Christ, sie sollen Dich so, wie Du bist, nehmen oder verlieren. Wenn der Geist der Menschen Dir eng erscheint, dann sage Dir, dass das Land Gottes weit ist und weit seine Hände, weit sein Herz. Zögere niemals, jenseits aller Meere, jenseits aller Grenzen, aller Vaterländer, aller Glaubenslehren das Weite zu suchen.

Was mich angeht, so habe ich das Ende meiner Reise erreicht. Vier Jahrzehnte Abenteuer haben meinen Schritt und mein Schnaufen schwerer gemacht. Ich habe kein anderes Begehren mehr als inmitten der Meinen lange friedvolle Tage zu leben. Und unter all jenen, die ich liebe, der Erste zu sein, der geht. Zu diesem Letzten Orte, wo nichts fremd ist im Angesichte des Schöpfers. ${ }^{10}$

Literatur ist Aufschub, ist immer zeitliche Distanz und zugleich ein Herausschieben des Endes, ein immer letztes Wort, das noch vom Überlebenswillen und Überlebenswissen kündet. So auch hier, gleich zu Beginn dieser letzten Seite, nachdem der Roman ,eigentlich' schon mit dem Sacco di Roma zu Ende gegangen war. Aber schreiben, noch einmal schreiben: Die Seite ist letzte Lebensbilanz, letztes Aufschreien vor dem Vergessen-Werden und ein letzter Verweis, dass der gesamte Text letztlich einem antiken Schema zu entsprechen vermag: einer Abfolge von Schiffbrüchen mit Zuschauer. ${ }^{11}$ Nichts könnte an diesem beweglichen Ort, an dem die Karawanen in die Karavellen übergegangen sind, das Leben von Léon l'Africain vor dem Hintergrund all der Städte, die er brennen und untergehen gesehen hat, besser zusammenfassen.

Zugleich wird in dieser Passage der eigene Sohn als Hauptzuschauer der Schiffbrüche hin befragt, auf ein Leben, das zwischen Muslimen, Juden und Christen immer wieder neuem Anpassungsdruck ausgesetzt sein wird, immer neuen Inquisitionen und Glaubenskongregationen, die alles durchwühlen und keinen Stein auf dem anderen lassen. Das Ende der Reise ist hier das des Buchs und zugleich ein Neuanfang im Leben eines anderen, der in der einen Diegese der

10 Maalouf, Amin: Lèon l'Africain, S. 349.

11 Vgl. Blumenberg, Hans: Schiffbruch mit Zuschauer. Paradigma einer Daseinsmetapher. Frankfurt am Main: Suhrkamp 1979. 
Sohn, in der anderen aber der Leser ist, dem sozusagen noch in letzter Sekunde einiges Weiteres an Lebenswissen - an LebensMitteln ${ }^{12}$ für die weitere Lebensreise - kommuniziert und mitgegeben werden soll.

Noch einmal wird das ständige Umherirren literarisch in Szene gesetzt, erscheint die Bilanz des Reisenden vor dem Antlitz Gottes in Form eines Lebensbuches, das dem Schöpfer am Ausgang überreicht wird. In dieser Schlusspassage, welche die Überfahrtsfiktion des Anfangs wieder aufnimmt, erscheint der „Créateur“ gleichsam selbst am Ende des Texts: Sein Name beschließt den Roman. Und eröffnet zugleich eine Art Traum, die Vision eines Paradieses, in dem es keine Fremden gibt, wo keiner wegen einer wie auch immer gearteten Differenz verfolgt oder auch nur befragt und unter Druck gesetzt wird.

Dieses angerufene Reich wird das Reich dieses „Schöpfers“ sein, aber in einem doppelten Sinne: des Schöpfers im monotheistischen Sinne, dem der Muslime, Juden und Christen, aber auch des Schöpfers im literarischen Sinne, wurde doch der Begriff „Créateur“ im Verlauf des 19. Jahrhunderts einem Prozess der Entsakralisierung ausgesetzt, der diese Bezeichnung für den Dichter und den Schriftsteller erreichbar machte. Der „Créateur“ ist folglich Gott, aber auch der Schriftsteller-Gott inmitten seiner Schöpfung. Dies also ist nicht nur das Paradies der Religion, sondern vielleicht weit mehr noch das der Literatur: Sie schafft jenen Raum, in dem niemand sich als „étranger“, als Fremder, vorkommen und fühlen muss. Dass wir uns bei dieser Schlusspassage in einer doppelten Diegese befinden, in welcher das Schlusswort eine doppelte Bedeutung besitzt, ist eine wundervolle Gabe des Erzählers und vor allem des Autors Amin Maalouf an seine Leserschaft!

In einem im Jahr 1997 geführten Interview mit dem Magazine littéraire hat Amin Maalouf einiges zu seiner Beziehung zum Libanon und zur wichtigen Rolle des Krieges in seinem literarischen Schaffen gesagt - auch zu jenem Krieg, der am 13. April 1975 praktisch unter dem Fenster seiner Wohnung begann. All dies ist für uns von größter Wichtigkeit, um die Schlussprojektion von Léon l'Africain in einem doppelten Licht noch besser verstehen zu können. Hier also ein kurzer Auszug aus dem Interview:

Ich bin dem Libanon als Land, aber vor allem als Symbol sehr verbunden, weil ich denke, dass es ein Land ist, welches den Versuch unternommen hat, verschiedene Gemeinschaften zusammenleben zu lassen, und dies ist für mich etwas sehr Wichtiges. [...] Der Libanon war auf alle Fälle das fortgeschrittenste Land der Region, und heute ist es weit von dieser einstigen Situation entfernt. Es war das Land der Toleranz. [...]

12 Vgl. Ette, Ottmar / Sánchez, Yvette / Sellier, Veronika (Hg.): LebensMittel. Essen und Trinken in den Künsten und Kulturen. Zürich: diaphanes 2013. 
Ich gehöre einer Minderheit an. In der Minderheit zu sein, ermutigt einen, eine universellere Vision zu haben, vor allem heute, wo wir in einer Welt leben, in welcher alle Kulturen in gewisser Weise in der Minderheit sind. Mit Hilfe eines Essays oder eines Romans über die Minderheitenposition in einer Gesellschaft und globaler noch über die Gesellschaften nachzudenken, in denen Minderheiten leben, legt ein Nachdenken darüber nahe über das, was Demokratie ist, über das, was die Welt von heute ist, und über das, was die Welt von morgen sein wird. [...]

Ich schrieb bereits, aber die Tatsache, wegen eines Krieges brutal alles stehen und liegen lassen zu müssen und mich in einem anderen Land wiederzufinden, dem einen wie dem anderen anzugehören, aber keinem von beiden ganz, wurde für mich wahrscheinlich zu einem entscheidenden Element. Und mich in diese höchste Marginalität zu werfen, welche das Schreiben von Fiktion ist, war ein Rettungsbalken. Stück für Stück habe ich den ganzen Rest abgelegt, habe quasi das Leben eines Eremiten gewählt und werde damit weitermachen, weil ich denke, dass meine Heimat das Schreiben ist. ${ }^{13}$

Wir finden in dieser langen Interviewpassage eine Vielzahl an Elementen wieder vor, die uns bereits aus Léon l'Africain bekannt sind, insofern sie als Autobiographeme oder essayartige Stücke in die Fiktion des Romans eingearbeitet wurden. Da sind zum einen Wunsch und Begehren, das Zusammenleben, das „vivre ensemble“, zu leben und zu erleben, und aus der Frustration dieses Begehrens der Wunsch, zumindest in der Literatur, in der Fiktion ein ZusammenLebensWissen zu entfalten, wie es in der Tat im Zentrum der Romane Amin Maaloufs steht. Immer wieder geht es bei diesem libanesisch-französischen Schriftsteller um Formen, Normen und Fragen des Zusammenlebens in Differenz - und warum dieses Zusammenleben an Grenzen stößt oder gar unmöglich wird. So erst konnte die Fiktion bei diesem ZwischenWeltenSchreiben zur eigentlichen, zur wahren Heimat dieses im Libanon geborenen und den Libanon vermissenden Literaten werden.

Innerhalb dieses komplexen ZwischenWeltenSchreibens ist als ein wesentlicher zweiter Punkt die Sprache anzusehen, die Amin Maalouf benutzt, also jenseits seiner arabischen Muttersprache das Französische. Es handelt sich dabei um eine Sprache, die Maalouf in seinem Französischen Exil spricht, die er aber schon - nach eigenem Geständnis - früh, vor allem abends bei der Niederschrift persönlicherer Dinge und zu einem Zeitpunkt benutzte, als er noch im Libanon auf Arabisch für eine große Beiruter Tageszeitung schrieb. Dieses translinguale, sich jenseits der eigenen Muttersprache ansiedelnde Schreiben beinhaltet ständige Reflexion, die aus dem Bereich ununterbrochenen Erlebens eines Lebens (und dann Schreibens) zwischen den Welten, zwischen Orient und Okzident stammt.

13 Maalouf, Amin, Interview mit François Bénichou: Amin Maalouf: ,Ma patrie, c’est l'écriture (interview)‘. In: Magazine littéraire 359 (novembre 1997), S. 114 f. 
Léon l'Africain ist ein wunderschönes literarisches Beispiel für das Schreiben in einem Bewegungs-Raum zwischen den Welten, welches sowohl den Protagonisten dieses Romans als auch seinen Verfasser prägt.

Es ist eine bemerkenswerte Formulierung Maaloufs, dass sein Schreiben seine eigentliche Heimat, seine „Patrie“ darstelle, ein Schreiben, das nicht in der Muttersprache - gleichsam in der „Matrie“ - verfasst ist, dabei aber die Sehnsucht nach einer Konvivenz erkennen lässt, in welcher entweder alle oder niemand ein Fremder oder eine Fremde ist. Dies zeigt uns einen Autor, der die Frage transarealer und transkultureller Beziehungen aus einer Perspektive untersucht und repräsentiert, die zutiefst von einer translingualen Position geprägt ist. Amin Maalouf bezog damit eine Stellung, welche vom beständigen Wechsel zwischen den Sprachen und dem Rückgriff auf eine später erlernte Literatursprache geformt ist, eine Position, in welcher die Bewegung quer zu den Sprachen, zu den Kulturen, stets auch in anderen diegetischen Zusammenhängen die Positionen seiner Protagonistinnen und Protagonisten bestimmt. Der libanesisch-französische Schriftsteller entfaltete damit seit den achtziger Jahren ein ZwischenWeltenSchreiben, welches in der aktuellen Literaturszene heute natürlich bei weitem nicht mehr derart ,minoritär‘ ist, wie es in den achtziger Jahren, zu Beginn der vierten Phase beschleunigter Globalisierung, noch erschien. 